\title{
Multi-dimensional characterization of mixed ligand nanoparticles using small angle neutron scattering
}

\author{
Zhi Luo ${ }^{\dagger *}$, Ye Yang ${ }^{\star}$, Aurel Radulescu ${ }^{\S}$, Joachim Kohlbrecher ${ }^{\perp}$, Tamim A. Darwish $^{`}$, Quy Khac Ong ${ }^{\dagger}$, \\ Stefan Guldin ${ }^{\dagger}$, Francesco Stellacci ${ }^{\dagger \nabla *}$ \\ ${ }^{\dagger}$ Institute of Materials, École Polytechnique Fédérale de Lausanne, 1015 Lausanne, Switzerland. \\ ¥ Department of Chemical Engineering, University College London, WC1E 7JE London, UK. \\ $\S$ Jülich Centre for Neutron Science (JCNS) at Heinz Maier-Leibnitz Zentrum (MLZ), Forschungszentrum Jülich GmbH, 85748 \\ Garching Germany. \\ ${ }^{\perp}$ Laboratory for Neutron Scattering and Imaging, Paul-Scherrer Institute, 5232 Villigen, Switzerland. \\ 'The National Deuteration Facility, Australian Nuclear Science and Technology Organisation, Lucas Heights, NSW, Australia. \\ $\nabla^{\nabla}$ Institute of Bioengineering, École Polytechnique Fédérale de Lausanne, 1015 Lausanne, Switzerland. \\ *Correspondence to: zhi.luo@pharma.ethz.ch and francesco.stellacci@epfl.ch
}

\begin{abstract}
The properties of ligand protected gold nanoparticles are determined by the synergistic interplay of their structural components, including the metal core, the ligand shell, and its solvation. However, the simultaneous characterization of all these components remains a major challenge given their distinctly different chemical nature. In the case of mixed ligand nanoparticles, this task becomes daunting due to the presence of intercorrelated additional parameters such as the ligand ratio, ligand spatial distribution, and the solvation of the heterogeneous ligand shell. Here we show that small angle neutron scattering (SANS) is a tool capable of simultaneously characterizing the core and the ligand shell of monodisperse mixed ligand gold nanoparticles. We systematically examine how each parameter (e.g. the core size, the thickness and composition of the ligand shell, and the mixed ligand spatial heterogeneity) affects nanoparticles' scattering profile. Quantitative information on these parameters is retrieved using analytical fitting as well as 3D modelling. Importantly, we show that SANS can evaluate the solvation degree of mixed ligand protected gold nanoparticles, a challenging task for any other characterization methods.
\end{abstract}

Ligand protected gold metal nanoparticles (NPs) are coreshell hybrid materials with unique structure-property relationships ${ }^{1}$. The metal core of NPs is known to determine their optical and electronical characteristics ${ }^{2-4}$, while the ligand shells play an important role on their interfacial properties ${ }^{5-9}$. A growing number of studies have pointed out that the contributions of the metal core and the organic shell to properties are cooperative $\mathrm{e}^{10-13}$. This phenomenon is magnified for mixed ligand protected nanoparticles (MNPs). For example, the size and shape of the gold core influence the spatial organization of ligand molecules on the surface ${ }^{14,15}$, which in turn can modify the optical properties of the metal core ${ }^{11,16}$. Furthermore, the interactions between NPs and biomolecules such as proteins and lipid membranes are a delicate interplay involving both the core size and the mixed ligand composition and morphology ${ }^{12,13,17-20}$.

A comprehensive and simultaneous characterization of various structural aspects for MNPs, e.g. the core sizes, the thickness, composition and morphology of the ligand shell, is therefore of great importance ${ }^{21-24}$. However, most of the existing characterization techniques for NPs only focuses on single component and treats their metallic and organic parts separately. There are several reasons for the lack of a universal technique. The first and most important one relates to the highly different chemical nature of the core, the ligands and the solvents. For gold NPs, the electron rich metal core allows precise characterization by electron microscope and X-ray scattering ${ }^{25,26}$. However, it is challenging for both techniques to probe the molecularly thin layer of organic molecules on the metal surface ${ }^{27}$. Similarly, approaches to characterize the ligands typically come from analytical methods developed for organic materials such as nuclear magnetic resonance (NMR), Fourier-transform infrared spectroscopy (FTIR), Raman spectroscopy, matrix-assisted laser desorption/ionization (MALDI) mass spectrometry and electron paramagnetic resonance (EPR) and have little interaction with the metallic core (that sometimes is even detrimental to the signal) ${ }^{28-31}$. It is even more challenging to characterize the spatial distribution of mixed ligand molecules co-assembling on the NP surfaces ${ }^{21,32}$. In such cases, various types of nanodomains (stripe-like, patchy and Janus) can form, which requires both spatial and chemical resolution for a proper characterization ${ }^{33-36}$. Currently, many characterization methods have to be combined to achieve a holistic understanding of all structural components. For example, a common practice is the combination of transmission electron microscopy (TEM) for the determination of metal core size together with NMR, MALDI mass spectroscopy or scanning tunneling microscope (STM) for the ligand 
shell ${ }^{31,34,37-41}$. However, while microscopic techniques image a few NPs with high resolution, spectroscopic methods such as NMR and MALDI mass spectrometry measure the ensemble average over the bulk sample $\mathrm{e}^{31,38,39}$. Therefore, due to the diverse working principles of the techniques, the integration of the results is not trivial. Moreover, beyond the structural elements of the NP itself, its interaction with the surrounding media such as solvents adds another level of complexity to interpret the macroscopic behavior of $\mathrm{NPs}^{42-44}$. The solvation degree of MNPs quantifies the volume fraction of solvent molecules inside the ligand shell, which indicates the favorable interactions between the NPs and solvents and leads to the stability of the NPs solution. Even fewer methods are capable of examining the solvation degree of MNPs in solution ${ }^{42,45}$. A method that could simultaneously measure these various structural aspects could therefore greatly benefit the field.

Small angle neutron scattering (SANS) is a very powerful technique for the characterization of the multicomponent coreshell NPs ${ }^{46-48}$. There are several studies that have utilized SANS to characterize different structural aspects of ligand shell protected gold nanoparticles ${ }^{49-55}$. For example, Diroll et. al. demonstrated that both the dimension of the core and ligand shell could be quantified for various types of homoligand gold nanoparticles (HNPs) $)^{51}$. Thanks to the difference in sensitivity of neutron and X-ray to organic and inorganic components, the combination of these two techniques gives insights on the deformability of the NPs. Kitchens and co-workers have shown that it is possible to monitor the structural change of the ligand shell on HNPs in response to anti-solvents using SANS $^{50}$. Furthermore, as the scattering pattern is very sensitive to the shape and symmetry of an object, SANS has been used to study the phase separation behavior of mixtures of small molecules or of polymers on gold nanoparticles ${ }^{53,55}$. The spatial distribution of mixed surfactant molecules on gold nanorods could be analyzed and related to the synthetic pathways ${ }^{55}$. Recently, some of us reported that SANS can probe the morphology of mixed ligand nanoparticles when combined with Monte Carlo type modelling ${ }^{37,40}$. The reconstructed $3 \mathrm{D}$ models could be further used to extract quantitative information and compared with other techniques, such as molecular dynamic simulations and mass spectrometry ${ }^{37,38}$. In summary, SANS has been explored as a powerful method to characterize the ligand shell of NPs. Nevertheless, no comprehensive methodology exists that allows to interpret all the information contained in the SANS and explore its capabilities in the simultaneous characterization of the core, the ligand shell, and the solvation of NPs, especially MNPs.

Here, we demonstrate the wide range of structural information that can be accessed by SANS. First, by comparing SANS data from various NPs, we show that SANS can distinguish NPs that differ only a few $\AA$ in their size, or only a few molecules in their ligand shell composition. Such sensitivity is comparable to that of TEM and ${ }^{1} \mathrm{H}$ NMR techniques. Through analytical fitting, it is also possible to quantify the solvation degree of MNPs, a challenging task for any other methods. Furthermore, the ability of SANS to capture ligand shell heterogeneities is explored. In summary, we establish here how SANS measurements can be used to meet a full spectrum of characterization requirements for MNPs covering a broad range of structural features and length-scales.

\section{EXPERIMENTAL SECTION}

Materials and Instruments. Deuterated alkanethiols 1butanethiol (BT-d), 1-octanethiol (OT-d) and 1-dodecanethiol (DDT-d) as well as 3-mercaptopropionic acid (MPA-d) ligand were purchased from CDN Isotopes, Inc. Deuterated 11mercaptoundecanoic acid (MUA-d) ligand is kindly provided by the National Deuteration Facility at the Australian Nuclear Science and Technology Organisation (ANSTO). All other chemicals were purchased from Sigma-Aldrich and used as received. TEM images were taken using FEI Tecnai Osiris machine. TGA measurements were performed using the TGA 4000 instrument from Perkin Elmer.

Nanoparticle synthesis and purification. Mixed ligand gold NPs were synthesized using the Stucky protocol ${ }^{56}$ followed by ligand exchange reaction as reported previously ${ }^{57}$. First, homo-alkanethiol protected NPs were synthesized as following: $123 \mathrm{mg}$ triphenylphosphinegold (I) chloride and 0.25 mmol alkanethiol ligand (e.g. DDT) were dissolved in the 20 $\mathrm{ml}$ chloroform and $20 \mathrm{ml}$ toluene mixtures. The solution was heated to $70{ }^{\circ} \mathrm{C}$ for 10 minutes before $217 \mathrm{mg}$ borane $\mathrm{t}$ butylamine complex was added under rapid stirring. The reaction proceeded for $1 \mathrm{~h}$ before cooling down to room temperature. Excess amount of methanol $(50 \mathrm{ml})$ was added to quench the reaction and precipitate the NPs. Black pellets of gold NPs were first collected by centrifugation (Thermo Fisher Sorvall ST 16R, at 4000 RPM) and then re-dispersed in $40 \mathrm{ml}$ anti-solvent, acetone, using vortex mixer (IKA) at fully speed. The vortex mixing was performed for more than 3 minutes in order to make sure that the pellets were broken into small pieces. The suspension was then precipitated using centrifugation at 4000 RPM. The anti-solvent purification step was then repeated for 2 times using acetone and 2 times using methanol. The final solid product was dried in vacuum overnight. A typical synthesis yielded $40 \mathrm{mg}$ gold NPs, corresponding to $90 \%$ conversion in terms of gold element.

The ligand exchange reaction was performed using the following protocol: $30 \mathrm{mg}$ of DDT or OT protected gold NPs were dissolved in $15 \mathrm{ml}$ chloroform. Different amount exchanging ligand molecules were added to the solution under stirring at room temperature. Specifically, for MPA-OT NPs, $3 \mathrm{mg}$ of MPA molecules were used. The reaction was quenched at $0.5 \mathrm{~h}, 3 \mathrm{~h}$ and $10 \mathrm{~h}$ respectively in order to achieve different ligand ratios, i.e. around MPA: OT $=1: 9,1: 4$ and 1: 1.5. For MPA-DDT NPs, the reaction time is adjusted to be 12 hours yielding MPA: DDT $=1: 1.5$. For MUA-OT NPs, $5 \mathrm{mg}$ of MUA ligand was added into the chloroform solution of gold NPs (30mg), the reaction proceeded 12 hours in order to achieve a ligand ratio of MUA: OT around 1:1. After the exchange reaction, nanoparticles formed suspensions featuring black color instead dark red indicating the solubility change that the NPs become insoluble in chloroform (except the MPA: OT = 1:9 sample, which is still soluble in chloroform). Excess amount of hexane $(20 \mathrm{ml})$ was added in order to fully precipitate the nanoparticles. The black precipitate was collected using centrifugation at 4000 RPM. The NPs were then re-dispersed using hexane and chloroform mixture (1:1) to form suspensions with the help of vortex mixer before centrifugation at 4000 RPM. The washing procedure was repeated for 3 times before the black pellets were dried under vacuum overnight. A typical ligand exchange reaction yielded $26 \mathrm{mg}$ gold NPs, corresponding to $87 \%$ yield.

NMR measurements. ${ }^{1} \mathrm{H}$ NMR spectra were taken using Bruker $400 \mathrm{MHz}$ spectrometer using methanol-d as the solvent. 
In order to determine the ligand shell composition, the gold core was etched using iodine before ${ }^{1} \mathrm{H}$ NMR measurement. Briefly, a stock iodine methanol solution with a concentration of 20 $\mathrm{mg} / \mathrm{ml}$ was first prepared. Then $0.6 \mathrm{ml}$ of the stock solution was added to $5 \mathrm{mg}$ of gold nanoparticles in a glass vial. The vial was then capped, sealed with parafilm and sonicated for 10 minutes. The supernatant was then taken for ${ }^{1} \mathrm{H}$ NMR measurement.

SANS/SAXS measurements. SANS measurements were conducted on the KWS-2 at Jülich Center for Neutron Science and SANS-I instrument at Paul Scherrer Institute. Measurements were performed at $20{ }^{\circ} \mathrm{C}$, using $1.5 \mathrm{~m}$ sampleto-detector distance, at $5 \AA$ wavelength with a collimation setup of $6 \mathrm{~m}$ and a q range covering $0.04 \AA-1$ to $0.4 \AA-1$. The sample concentration was approximately $10 \mathrm{mg} / \mathrm{ml}$ corresponding to a volume fraction of gold nanoparticles solution of less than $0.1 \%$. The two-dimensional scattering data were processed and reduced using QtiKWS software at KWS2 and BerSANS software at PSI including radial averaging, background subtraction, empty cell and transmission correction, and normalization to an absolute scale. The choice of deuterated solvents was based on the solubility of different NPs. Specifically, for MPA-DDT NPs, THF-d was used as the solvent; for BT-OT-DDT NPs, the solvent was toluene-d; for MPA-OT NPs, the solvent is ethanol-d or a mixture of ethanol and ethanol-d to adjust the contrasts; for MUA-OT NPs, the solvent is THF-d.

SAXS data were recorded using SAXSLAB Ganesha $300 X \mathrm{~L}$ at $20^{\circ} \mathrm{C}$ with a $2 \mathrm{~mm}$ beam stop and a q range of 0.015 $\AA^{-1}$ to $0.65 \AA^{-1}$. Glass capillaries with outside diameter of $1 \mathrm{~mm}$ was used for the measurement. The solvent was the same as used in SANS measurements. Data reduction was performed using SAXSGUI software.

Data analysis. The SasView software (version 4.1) was used for the analysis of the SANS data of mixed ligand nanoparticles. Core-shell sphere models were used for the analytical fitting in order to obtain parameters such as the thickness and contrast of the ligand shell. During the fitting, the parameters for the size and contrast of the gold core was fixed as characterized from TEM and SAXS data. The P(r) function was calculated using the GNOM package in the ATSAS software ${ }^{58}$

For $a b$ initio fitting and 3D model reconstruction from the SANS data, the MONSA program ${ }^{59}$ in ATSAS software ${ }^{58}$ was used as described before ${ }^{37}$. In brief, a spherical search volume composed of close packed small beads with $2 \AA$ radius was generated according the $\mathrm{D}_{\max }$ value in the $\mathrm{P}(\mathrm{r})$ function. As the core radius is $28 \AA$, the beads within the central $24 \AA$ radius were fixed to gold in order to reduce the calculation time and act as physical constraints. Between 30 and $38 \AA$ radius, the bead assignments were limited to the MUA and DDT ligands or solvent, while All other beads were free to be assigned to all four possible components. Simulated annealing algorithm implemented in MONSA then searches for the best bead assignments, i.e. 3D model that fits simultaneously all the SANS curves and minimize the overall discrepancy between the experimental data and fits based on the theoretical spectra of the multiphase bead model. The fitting was run multiple times always starting from random bead assignments and the results were all similar.

\section{RESULTS AND DISCUSSION}

We first present an overview of SANS pattern for mixed ligand nanoparticles in solution. As shown in Figure 1, there are several different q-range regions in the SANS profile that are dominated by specific structural features of mixed ligand nanoparticles. The low q regions are affected mainly by the inter-particle interactions ${ }^{22}$. Figure $1 \mathrm{~A}$ represents the scattering of ideal non-interacting nanoparticles, which is the case for dilute samples. The structure factor of the sample equals to 1 as the scattering in the low angle region in the figure is rather flat and featureless, indicating an ideal gas like status of the particles (region 1). In contrast, Figure $1 \mathrm{~B}$ shows two examples of NPs with significant inter-particle interaction potential. When the ionic strength of the solvent is low and the repulsive interactions between the NPs are not screened, a broad peak could present in the region 1 as shown by the red curve, which corresponds to the concentration dependent mean nanoparticle distance $^{60}$. If the interaction potential is attractive, the black curve in Figure 1B shows that scattering intensity increases towards lower $\mathrm{q}$ angles. In both cases, it is challenging to retrieve the shape and size of the nanoparticles without proper knowledge of the structure factor. Therefore, all the NPs discussed below were measured in non-interacting conditions, achieved by choosing the suitable solvents and keeping the nanoparticle concentrations low.

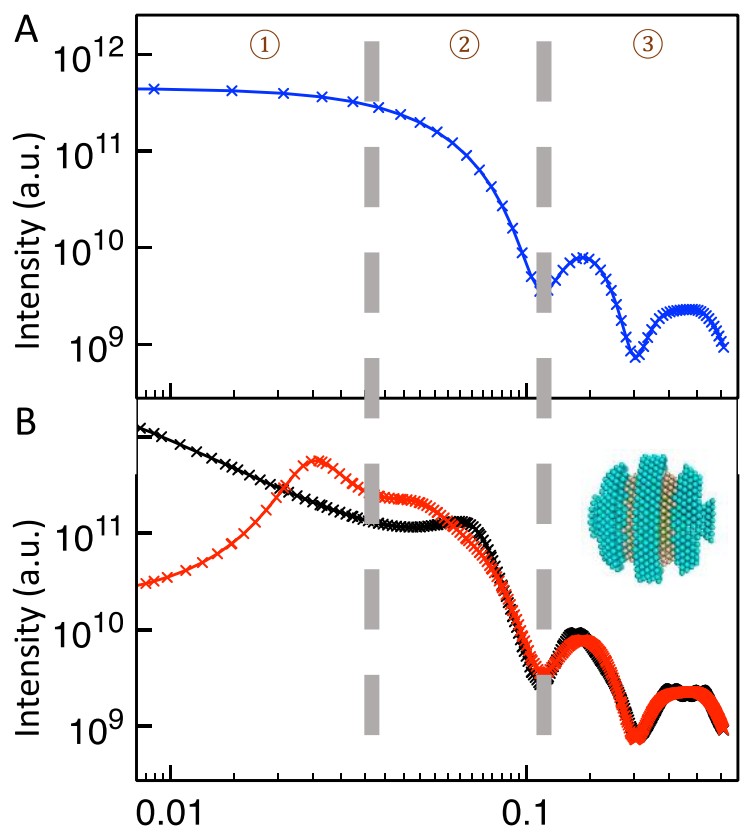

Figure 1. Theoretical SANS data of mixed ligand nanoparticles under different inter-particle interaction potential. (A) The scattering profile (blue curve) of non-interacting NP samples (form factor). The nanoparticle model has a core diameter of 5 $\mathrm{nm}$ and presents stripe-like morphology in the ligand shell. (B) SANS pattern of NPs with attractive (black) and repulsive (red) inter-particle interactions. The inset cartoon represents the idealized stripe-like NP model that was used to calculate the SANS curves. All the theoretically calculated data in this work are plotted using cross symbols.

The intermediate and q region (named 2 and 3 in Figure 1) is the focus of the current study, from which detailed information on the nanoparticle form factors could be extracted. As discussed below, we show that these two regions are affected by the composition of the ligand shell as well as the degree of 
solvation $^{61}$. For multicomponent ligand shells, the high $\mathrm{q}$ regions are further affected by the geometry of nanoscale phase separation of ligands. In the following sections, we will examine how each of the q region reveals different mixed ligand nanoparticles structures.

Dimensional Information. As the size of a NP is always the primary question in its characterization, we first focus on demonstrating the sensitivity of SANS to the dimension of both the metal core and organic shell. Two series of gold NPs were synthesized according to previous literature ${ }^{37,56,57}$. First, three MNPs with different core sizes but the same ligand shell coating were produced using a ligand exchange protocol ${ }^{56,57}$. TEM analysis (Figure S1) shows high degree of monodispersity for all three NPs with the core radius being $1.9 \pm 0.2 \mathrm{~nm}, 2.2 \pm 0.2$ $\mathrm{nm}, 2.7 \pm 0.3 \mathrm{~nm}$, respectively. The error bars represent the standard deviation of core radius based on the analysis of more than 500 NPs using ImageJ software. The metal core size as well as monodispersity were further confirmed with small angle X-ray scattering (SAXS) showing good agreement with the TEM results (Figure S2). All the nanoparticles were protected with a mixture of 3-mercaptopropionic acid (MPA) and 1dodecanethiol (DDT) with a ligand ratio of $40 \%$ : $60 \%$ as controlled by the ligand exchange time and reaction stoichiometry ${ }^{11,57}$. The ligand ratio was determined using ${ }^{1} \mathrm{H}$ NMR after etching the gold core using iodine ${ }^{28,62}$, as shown in Figure S3. Ligand densities were calculated to be $13 \%, 11 \%$ and $8 \%$ for the $1.9 \mathrm{~nm}, 2.2 \mathrm{~nm}$ and $2.7 \mathrm{~nm}$ nanoparticles, respectively, as calculated from TGA data.
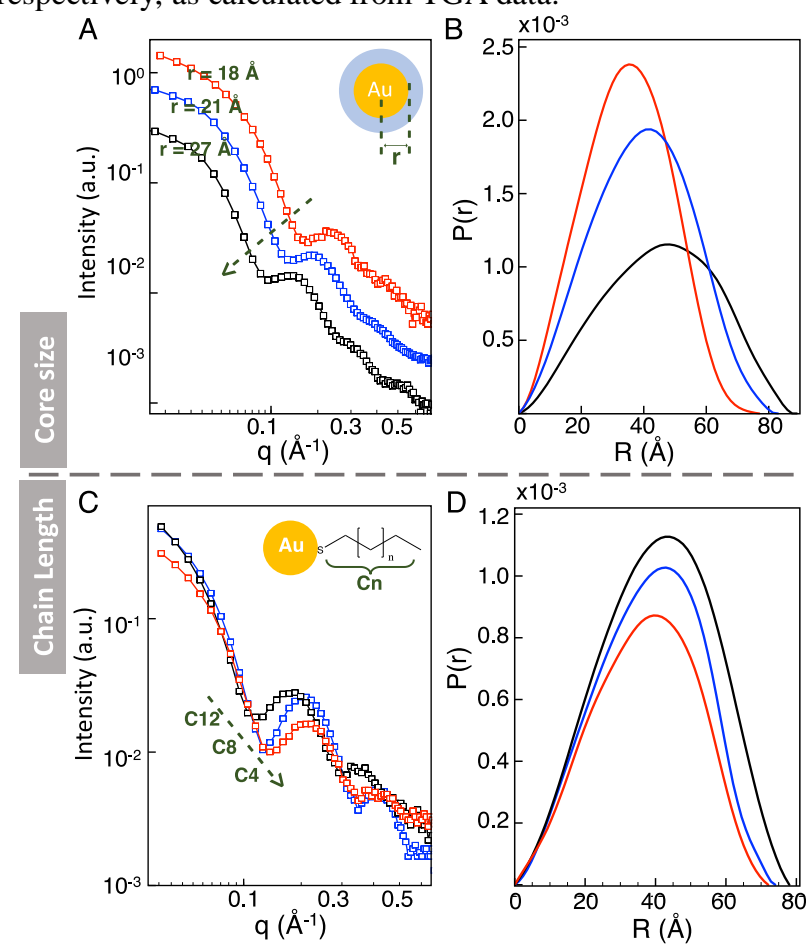

Figure 2. SANS data of mixed ligand nanoparticles with different core sizes and ligand chain length. (A) SANS data and (B) corresponding P(r) data of three MPA-DDT NPs with the same ligand shell but different core sizes, i.e. $1.8 \pm 0.2 \mathrm{~nm}, 2.1$ $\pm 0.2 \mathrm{~nm}, 2.7 \pm 0.2 \mathrm{~nm}$ based on fitting using a core-shell spherical model. The solvent is THF-d for these NPs. The intensities of the three data were scaled for the sake of clarity. (C) SANS data and (D) corresponding P(r) data of three nanoparticles with same core size and ligand shell composition but highlight molecules of different chain length. The solvent is toluene-d for these NPs. The red curve stands for the nanoparticles with both DDT and OT ligands deuterated; the blue curve stands for the nanoparticles with both DDT and BT ligands deuterated; the black curve stands for the nanoparticles with both OT and BT ligands deuterated.

SANS data was recorded using deuterated tetrahydrofuran (THF-d) as solvent. As shown in Figure 2A, although the variations in the core size are less than $5 \AA$, the scattering profile of the nanoparticles are clearly different. The position of the oscillation valley shifts to lower q values with the increase of the core radius. The same trend is also clearly shown in the pair distance distribution functions, $\mathrm{P}(\mathrm{r})$, calculated using GNOM package $^{58}$, see Figure $2 \mathrm{~B}$. The $\mathrm{D}_{\max }$ values in $\mathrm{P}(\mathrm{r})$, the maximum distance in the scattering object, indicate that the overall diameter of the three nanoparticles are $77 \AA, 83 \AA$ and $89 \AA$, respectively. The shape of the black curve in $\mathrm{P}(\mathrm{r})$ function deviates from the bell shape. This could be due to the specific combination of ligand length and composition as well as spatial distribution of the ligands deviating from random mixing. However, it is difficult to directly correlate the peak in $\mathrm{P}(\mathrm{r})$ to structural features in this case. The SANS data were further fitted with a core-shell sphere model using SasView software in which the shell thickness, solvent and core contrasts were fixed. The fitted core radius results agree very well with the TEM analysis being $1.8 \pm 0.2 \mathrm{~nm}, 2.1 \pm 0.2 \mathrm{~nm}$, $2.7 \pm 0.2 \mathrm{~nm}$, respectively. While the spatial resolution of SAXS on NPs has been reported to reach sub- $5 \AA^{61}$, the resolution of SANS data is often compromised due to the smearing effects such as neutron wavelength spread, aperture size, detector pixel sizes, etc ${ }^{63}$. Nevertheless, here we confirm that similar to SAXS, SANS can also efficiently distinguish NPs with core radius differences of less than $5 \AA$, a dimension comparable to the diameter of a single gold atom.

The second series of mixed ligand NPs feature the same core size $(2.7 \pm 0.3 \mathrm{~nm}$, as confirmed with TEM and SAXS shown in Figure S1 and Figure S2). A mixture of three different alkanethiol ligands were used to co-protect the NPs, i.e. 1butanethiol (BT), 1-octanethiol (OT) and DDT. On each of the $\mathrm{NP}$, two of the three ligands were deuterated in order to match the contrast of the solvent (toluene-d), while the other ligand was non-deuterated and thus has large negative contrast. It has been shown previously that ligand deuteration does not affect the composition as well as the spatial heterogeneity of the ligand shell ${ }^{37}$. Three mixed ligand NPs were synthesized using a modified Stucky's protocol followed by ligand exchange under the same synthetic protocol (e.g. temperature, solvent, reagent concentration and time) to make sure that they have the same core size and ligand shell composition. As shown in Figure 2C, clear differences in the scattering profiles of the three nanoparticles could be captured. The oscillation valley shifts to higher q with the decrease of the overall alkane chain length of unmatched ligand molecules, indicating that the size of the NPs become smaller. The P(r) plot, Figure 2D, confirms the trend with the $D_{\max }$ values being $72 \AA, 74 \AA$ and $78 \AA$ for BT, OT and DDT highlighted NP respectively. Core-shell model fitting indicates that the thickness of the BT, OT and DDT ligand layers are $6.9 \pm 1.0 \AA, 8.8 \pm 0.9 \AA, 12.2 \pm 1.2 \AA$, respectively. Such chain length profile agrees quantitatively with the previous reports ${ }^{37,50,51}$ and again highlighting the sensitivity of SANS on the monolayer of ligand molecules down to only a few $\AA$. 
Compositional Heterogeneity and Solvation. The next important parameter for MNPs is the ratio of the two different ligand molecules. Many important interfacial properties of the NPs are determined by the surface composition, such as the solubility ${ }^{64}$, interfacial energy ${ }^{65,66}$, sensing and interaction with biomolecules ${ }^{5,20}$. Currently the most accurate way to determine the ligand ratio is ${ }^{1} \mathrm{H} \mathrm{NMR}^{21}$. Recent studies also suggest that MALDI-TOF mass spectrometry is an alternative way of determining ligand ratio $^{38,67,68}$. Unfortunately, both methods have limitations. For ${ }^{1} \mathrm{H}$ NMR analysis, the core of the NPs needs to be decomposed using harsh chemical or thermal treatments to release ligand molecules ${ }^{28}$, while MALDI-TOF analysis may have difficulties once the molecular weight differences between the two molecules are too large or too small. On the contrary, SANS measures the intact NPs in the native solution and can be applied to ligands of varying chain lengths as demonstrated above. By selectively deuterating one of the ligand molecules, one can generate large difference in the contrasts of the two ligands. Importantly, as the ligand shell contrast also depends on the degree of solvation ${ }^{50}$, by varying the contrast of solvent (deuteration content), it is possible to quantify the solvation amount together with ligand ratio at the same time.
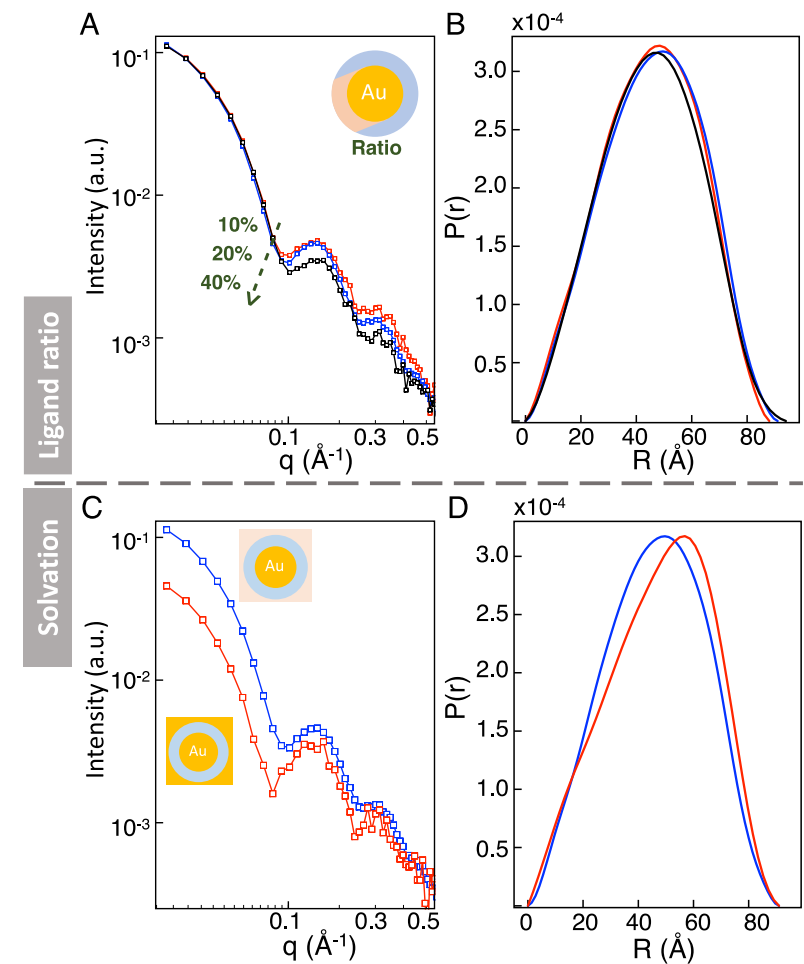

Figure 3. SANS data of mixed ligand nanoparticles with varying ligand ratio and solvation. (A) SANS data and (B) corresponding $\mathrm{P}(\mathrm{r})$ functions of MPA-d and OT mixed ligand protected nanoparticles. The ratio of MPA-d: OT are $l_{M P A}: l_{O T}=1: 9$ (red), $1: 4$ (blue) and 1:1.5 (black), respectively. (C) SANS data and (D) P(r) functions of 20\% MPA-d mixed ligand NP under different solvent contrast conditions. The SLDs of ethanol solvents are $5.8 \times 10^{-6} \AA^{-2}$ for the blue curves and 4.6 x $10^{-6} \AA^{-2}$ for the red curves.

To first examine the effect of ligand ratio on the SANS profile of NPs, three gold NPs protected with deuterated MPA
(MPA-d) and OT with varying ligand ratios were prepared. The ligand exchange protocol was adopted to control the NPs with the same core size: $2.9 \pm 0.3 \mathrm{~nm}$ in radius as measured by TEM and SAXS, Figure S1 and S2. To determine the ligand shell composition, NPs composed of non-deuterated ligands with exactly the same synthesis procedure were synthesized. The MPA: OT ratios were measured by ${ }^{1} \mathrm{H}$ NMR after iodine decomposition (Figure S3) to be $l_{M P A}: l_{O T}=1: 9,1: 4$ and 1: 1.5, respectively. As shown in Figure $3 \mathrm{~A}$, the higher the surface fraction of MPA-d ligands, the lower the intensity at high q. This is due to the fact that the scattering length density of MPA-d is close to that of the solvent resulting in less contrast and hence lower scattering intensity. Furthermore, the $\mathrm{P}(\mathrm{r})$ profile shows that the $D_{\max }$ values slightly decrease with increasing fraction of MPA-d due to the chain length differences of the two ligands.

In order to quantify the ligand ratio and solvation using SANS, we performed the measurements on the same NP under two different contrast conditions. The scattering length density (SLD) of ethanol solvent $\left(\rho_{\text {sol }}\right), 5.8 \times 10^{-6} \AA^{-2}$, matched that of the deuterated ligands. The second solvent contrast $\left(\rho_{\text {sol }}^{\prime}\right), 4.6$ $\mathrm{x} 10^{-6} \AA^{-2}$ was generated by mixing ethanol with deuterated ethanol to match the SLD of gold core. As shown in Figure 3C, significant changes in the scattering patterns under these two contrast conditions can be observed. The differences can be better rationalized from the $\mathrm{P}(\mathrm{r})$ function, Figure $3 \mathrm{D}$. As the gold core is contrast matched, the overall form factor of the NP is close to a hollow spherical shell. Therefore, while both the $\mathrm{P}(\mathrm{r})$ functions have bell shapes, the one with solvent-core contrast matching condition is more asymmetric with the peak position shifts to larger distances.

The contrast of the ligand shell has the following relationships with the volume fraction of the two ligands in the ligand shell and the degree of solvation $(\chi)$ :

$$
\rho_{\text {shell }}=\rho_{M P A} * f_{M P A}+\rho_{O T} * f_{O T}+\rho_{\text {sol }} * \chi
$$

Here, $f_{M P A}$ and $f_{D D T}$ are the volume fraction of MPA and OT ligands in the ligand shell respectively. $\rho_{M P A}$ and $\rho_{O T}$ are the contrast of the two types of ligands calculated from their chemical formula and density. A core-shell model is used to fit the scattering curve in order to calculate the overall ligand shell contrast, $\rho_{\text {shell }}$. By applying the second solvent contrast, one obtains a similar relation:

$$
\rho_{\text {shell }}^{\prime}=\rho_{M P A} * f_{M P A}+\rho_{O T} * f_{O T}+\rho_{\text {sol }}^{\prime} * \chi
$$

Hence two measurements in two solvents depend solely on three parameters, $f_{M P A}$ and $f_{O T}$, and $\chi$, yet only two of these parameters are independent, As the $\chi$ value is the volume fraction of solvents inside ligand shell, consequently the following relationship holds by definition:

$$
\chi=1-f_{M P A}-f_{O T}
$$

For example, from equations (1) and (2), it is straightforward to calculate the solvation value, $\chi$ and hence the $f_{M P A}$ and $f_{O T}$ :

$$
\chi=\frac{\rho_{\text {shell }}-\rho_{\text {shell }}^{\prime}}{\rho_{\text {sol }}-\rho_{\text {sol }}^{\prime}}
$$

At this point one has two measurements and two parameters, that can be readily derived. It should be noted that the volume fraction can be converted to the ligand ratio through the equation (5) and therefore can be readily compared to ratio derived from ${ }^{1} \mathrm{H}$ NMR measurement.

$$
f_{M P A}: f_{O T}=l_{M P A} * \frac{M_{W, M P A}}{d_{M P A}}: l_{O T} * \frac{M_{W, O T}}{d_{O T}}
$$


Both SANS curves under two solvent contrast conditions were fitted with the core-shell sphere model. During the fitting, the contrast of the gold core, solvent and the radius of the core were fixed allowing only the thickness of the ligand shell $\left(r_{\text {shell }}\right)$ and the ligand shell overall contrast $\left(\rho_{\text {shell }}\right)$ to be optimized. Table 1 shows a summary of all the resulted parameters from the fitting and calculations. Notably, the calculated ligand shell composition agrees well with the results from ${ }^{1} \mathrm{H}$ NMR, indeed when subtracting the percentage composition for one ligand derived from SANS to that derived from ${ }^{1} \mathrm{H}$ NMR one obtains a number whose absolute value is always lower than $10 \%$. Due to the longer chain length of OT than MPA, as the fraction of MPA ligands in ligand shell increases, the averaged thickness of the ligand shell, $r_{\text {shell }}$, decreases slightly. On the other hand, the degree of solvation, $\chi$, increases significantly from $15 \%$ to $23 \%$ as the fraction of MPA increases from $10 \%$ to $38 \%$. The increase in solvation volume is due to the fact that the MPA ligands are more polar than OT and thus are better solvated in the polar solvent, ethanol. The trend is also supported by the fact that NPs coated with OT molecule alone are not soluble in ethanol at all. It should be noted that it has been reported that the interfacial properties of nanoparticles coated with a mixture hydrophobic and hydrophilic ligands are very complex $^{10,66}$. For example, the solubility of the MPA-OT NPs deviates from monotonic dependence on composition in various solvents ${ }^{69}$. Similarly, the interfacial energy of NPs protected with a mixture of 6-mercaptohexanol and OT ligands features sizeable non-additive behavior ${ }^{10}$. To the best of our knowledge, quantifying the degree of solvation and correlating it with the composition of the ligand shell has not been reported before. The analysis method described here could thus bring new insights into the wetting phenomena of MNPs.

Table 1. List of SANS fitting results. The same nanoparticles were measured in two different solvent contrast $\left(\rho_{\text {sol }}\right)$ conditions and the contrasts of ligand shell $\left(\rho_{\text {shell }}\right)$ were obtained by fitting the SANS patterns using core-shell sphere model. The solvation parameters as well as ligand ratios were then calculated using the equations above.

\begin{tabular}{ccccccc}
\hline Sample & $r_{\text {shell }}(\AA)$ & $\rho_{\text {Sol }}\left(10^{-6} \AA^{-2}\right)$ & $\rho_{\text {shell }}\left(10^{-6} \AA^{-2}\right)$ & $\chi$ & $f_{M P A}$ & $f_{M P A}\left({ }^{1} \mathrm{H}\right.$ NMR) \\
$l_{M P A}: l_{O T}$ & 14.7 & 5.8 & 0.85 & $14.9 \%$ & $10.4 \%$ & $13 \%$ \\
$1: 9$ & & 4.6 & 0.69 & & & \\
\hline$l_{M P A}: l_{O T}$ & 14.5 & 5.8 & 1.51 & $20.9 \%$ & $17.4 \%$ & $24 \%$ \\
$1: 4$ & & 4.6 & 1.28 & & & \\
\hline$l_{M P A}: l_{O T}$ & 13.2 & 5.8 & 2.45 & $22.9 \%$ & $37.7 \%$ & $41 \%$ \\
$1: 1.5$ & & 4.6 & 2.19 & & & \\
\hline
\end{tabular}

Spatial Heterogeneity of Ligand shell. As it has been demonstrated recently, SANS combined with multiphase bead modelling can reconstruct various morphologies of mixed ligand structures in a quantitative way $^{37,40}$. While previous works have found that the oscillations of the form factor were related to the overall symmetry of the ligand organization, qualitative guidelines for the interpretation of the spectra have not been established yet. Here, to better understand this correlation, we first calculate and compare the theoretical SANS pattern of NPs with different idealized spatial distributions and nanodomain sizes. As shown in Figure 4, five idealized ligand organization geometries (Random, Janus, Patchy and different stripe thicknesses) were generated and their theoretical scattering profile was calculated using the SLD condition of the MPA-DDT nanoparticles. The SANS data were properly smeared using experimental parameters of KWS2 instrument ${ }^{70}$. Figure $4 \mathrm{~A}$ and $4 \mathrm{~B}$ show that while the four NPs have the same core sizes and ligand ratio, their scattering form factors are distinctively different. The low q regions of all the four scattering curves perfectly overlap up to $0.08 \AA^{-1}$, corresponding to the $\mathrm{D}_{\max }$ dimension $(80 \AA)$, confirming that the NPs have practically the same radius of gyration $\left(R_{g}\right)$. The differences in the high q regions therefore can only be ascribed to the ligand shell organization. In fact, for the stripe-like NPs with different stripe widths and random NP, the scattering curves overlaps up to $\sim 0.15 \AA^{-1}$, while the Janus NP gives a scattering profile that deviates the most from the rest. The scattering pattern of the NP with the thinnest MPA stripe width ( $\sim 6 \AA)$, shown in the blue curve in Figure $4 \mathrm{~A}$, is closest to a spherical scattering object. When the stripe width is $\sim 10 \AA$, the SANS profile of the NP (red curve) deviates significantly from the power law of spherical NP in the q range higher than $0.5 \AA^{-}$ 1. Therefore, the scattering form factors of these NPs are highly affected by the stripe-like domain sizes especially at high $\mathrm{q}$ angles. Considering that the overall radius of the NPs is around $50 \AA$, a difference of $5 \AA$ in stripe width would significantly affects the overall sphericity of the ligand shell. 

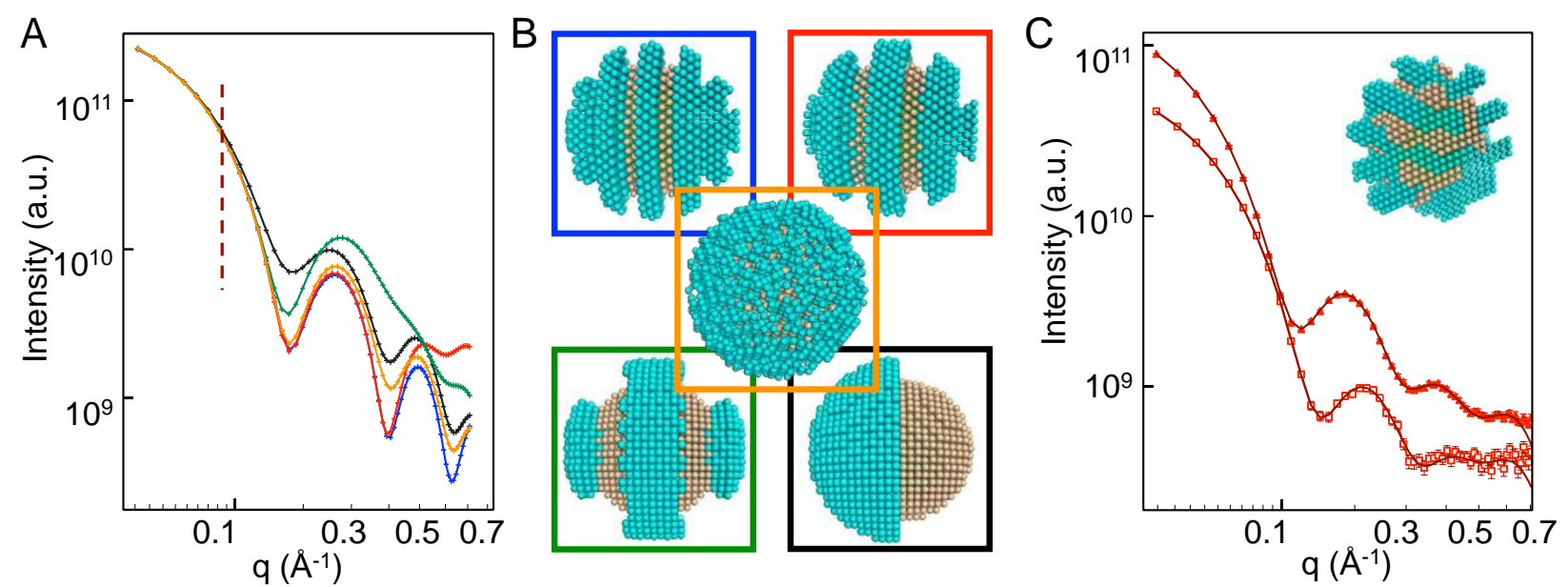

Figure 4. Effect of ligand shell organization on the scattering form factors of mixed ligand nanoparticles. (A) Theoretical S ANS pattern and (B) the corresponding morphologies of random, ideal stripe-like nanoparticles with different stripe width as well as Janus nanoparticles. The colors of the curves in (A) correspond to different models in (B). The smearing effects were taken into consideration when calculating the SANS data. In the theoretical SANS data in (A), the SLD of the solvent matches the longer ligand (DDT) in deuterated form while the shorter ligand has a contrast of hydrogenated ligand form. (C) Experimental SANS data (symbol) and the fit (full line) of MUA-OT nanoparticles. The triangle symbolled data stands for SANS measurements on MUA-(OT-d) nanoparticles; the square symbol is for (MUA-d)-OT nanoparticles. The inset shows the multiphase bead model generated using the MONSA program. The blue beads in the model correspond to the MUA ligands while yellow beads are OT ligands.

Based on the calculated SANS pattern of various morphologies, we have furthermore screened and compared a large amount of experimental data. In this way, similar scattering features of the theoretical idealized stripe models in the experimental data of some synthetic MNPs were found for the first time. For example, we synthesized gold NPs protected with 11-mercaptoundecanoic acid (MUA) and OT ligands. Two batches of NPs were prepared using each with one ligand deuterated and another hydrogenated, i.e. MUA-(OT-d) NP and (MUA-d)-OT NP. SANS data (Figure 4C) of the two NPs in THF-d solvent were recorded and thus in each scattering pattern the contrast of the deuterated ligands is matched with the solvent, highlighting the other ligand. The square symbolled curve in Figure 4C, corresponding to (MUA-d)-OT NP, shows high similarities with theoretical pattern of the thin stripe-like morphology (red curve in Figure 4A). This indicates that the OT ligands form thin stripes within the ligand shell. Multiphase bead model was then built by simultaneously fitting the two scattering curves using the MONSA program utilizing simulated annealing algorithm as described previously ${ }^{37,40}$. The inset in Figure 4C shows the bead model of the MUA-OT NP. The reconstructed model shows that both MUA and OT ligands form stripe-like domains with stripe thickness being around 10 $\AA$, which is determined as described previously using the averaged value of the distance between the two boundary lines in the MONSA model ${ }^{37}$. Indeed, both the structural features and dimension of the stripe-like domains in (MUA-d)-OT NP model resembles the model in Figure 4B. Furthermore, applying the same principle in the previous section, we could quantify the degree of solvation of the MUA-OT NP to be $22 \%$. Therefore, by combining analytical fitting with multiphase bead modelling, it is possible to extract both ligand shell spatial heterogeneity and solvation degree from the same set of SANS experiments.

\section{CONCLUSION}

We demonstrate here that SANS can act as a technique to simultaneously characterize various different structural aspects of MNPs. A combined analysis using analytical fitting and 3D multiphase bead modelling yields quantitative information about the dimension, composition and morphology of NPs with $\AA$ length scale and molecular accuracy. The contrast variation approach helps to highlight different components of ligand shells and allows for the extraction of characteristic length scales and compositions. There are still several limitations for the SANS including the requirement of deuterated ligands, access of the instrument as well as well as the data analysis procedures that are computing time demanding. Nevertheless, the method is versatile and almost without constraints on the size and morphology of the MNPs. Therefore, we believe that SANS could become an important technique in establishing the structure-property relationships of MNPs.

\section{ASSOCIATED CONTENT}

\section{Supporting Information}

The Supporting Information is available free of charge on the ACS Publications website.

TEM, SAXS and ${ }^{1} \mathrm{H}$ NMR characterization of mixed ligand nanoparticles. (PDF)

\section{AUTHOR INFORMATION}

\section{Corresponding Author}

* Email: zhi.luo@pharma.ethz.ch and francesco.stellacci@epfl.ch

\section{Author Contributions}

All authors have given approval to the final version of the manuscript.

\section{ACKNOWLEDGMENT}


Z.L. and F.S. gratefully acknowledge funding from the Swiss National Foundation Division II. Y.Y. acknowledges University College London for the Overseas Research Scholarship and the Graduate Research Scholarship and thanks Dr. Han Wu and the EPSRC CNIE research facility service (EPSRC Award, EP/K038656/1) at UCL for SAXS facility access. T.D. acknowledges the support from the National Collaborative Research Infrastructure Strategy - an initiative of the Australian Government. T.D. thanks Anwen Krause-Heuer for her help with the deuteration work. This work benefited from the use of the SasView application (http://www.sasview.org/), originally developed under NSF award DMR-0520547. SasView contains code developed with funding from the European Union's Horizon 2020 research and innovation programme under the SINE2020 project, grant agreement No 654000 .

\section{REFERENCES}

(1) Eustis, S.; El-Sayed, M. a. Why Gold Nanoparticles Are More Precious than Pretty Gold: Noble Metal Surface Plasmon Resonance and Its Enhancement of the Radiative and Nonradiative Properties of Nanocrystals of Different Shapes. Chem. Soc. Rev. 2006, 35 (3), 209-217.

(2) Burda, C.; Chen, X.; Narayanan, R.; El-Sayed, M. A. Chemistry and Properties of Nanocrystals of Different Shapes. Chem. Rev. 2005, 105 (4), 1025-1102.

(3) Sau, T. K.; Rogach, A. L.; Jäckel, F.; Klar, T. A.; Feldmann, J. Properties and Applications of Colloidal Nonspherical Noble Metal Nanoparticles. Adv. Mater. 2010, 22 (16), 1805-1825.

(4) Nakanishi, H.; Bishop, K. J. M.; Kowalczyk, B.; Nitzan, A.; Weiss, E. A.; Tretiakov, K. V.; Apodaca, M. M.; Klajn, R.; Stoddart, J. F.; Grzybowski, B. A. Photoconductance and Inverse Photoconductance in Films of Functionalized Metal Nanoparticles. Nature 2009, 460 (7253), 371-375.

(5) Mancin, F.; Prins, L. J.; Scrimin, P. Catalysis on GoldNanoparticle-Passivating Monolayers. Curr. Opin. Colloid Interface Sci. 2013, 18 (1), 61-69.

(6) Moyano, D. F.; Saha, K.; Prakash, G.; Yan, B.; Kong, H.; Yazdani, M.; Rotello, V. M. Fabrication of Corona-Free Nanoparticles with Tunable Hydrophobicity. ACS Nano 2014, 8 (7), 6748-6755.

(7) Lee, H. Y.; Shin, S. H. R.; Drews, A. M.; Chirsan, A. M.; Lewis, S. A.; Bishop, K. J. M. Self-Assembly of Nanoparticle Amphiphiles with Adaptive Surface Chemistry. ACS Nano 2014, 8 (10), 9979-9987.

(8) Zhou, J.; Ralston, J.; Sedev, R.; Beattie, D. A. Functionalized Gold Nanoparticles: Synthesis, Structure and Colloid Stability. J. Colloid Interface Sci. 2009, 331 (2), 251-262.

(9) Pengo, P.; Pasquato, L. Gold Nanoparticles Protected by Fluorinated Ligands: Syntheses, Properties and Applications. $J$. Fluor. Chem. 2015, 177, 2-10.

(10) Silvera Batista, C. A.; Larson, R. G.; Kotov, N. A. Nonadditivity of Nanoparticle Interactions. Science (80-. ). 2015, 350 (6257), 1242477-1242477.

(11) Goldmann, C.; Lazzari, R.; Paquez, X.; Boissière, C.; Ribot, F.; Sanchez, C.; Chanéac, C.; Portehault, D. Charge Transfer at Hybrid Interfaces: Plasmonics of Aromatic Thiol-Capped Gold Nanoparticles. ACS Nano 2015, 9 (7), 7572-7582.

(12) Walkey, C. D.; Chan, W. C. W. Understanding and Controlling the Interaction of Nanomaterials with Proteins in a Physiological Environment. Chem. Soc. Rev. 2012, 41 (7), 2780-2799.

(13) Stellacci, F.; Carney, R. P.; Van Lehn, R. C.; Irvine, D. J.; Alexander-Katz, A.; Yang, Y.; Atukorale, P. U. Effect of Particle Diameter and Surface Composition on the Spontaneous Fusion of Monolayer-Protected Gold Nanoparticles with Lipid Bilayers. 2013, 13 (9), 4060-4067.

(14) Singh, C.; Ghorai, P. K.; Horsch, M. a; Jackson, A. M.; Larson, R. G.; Stellacci, F.; Glotzer, S. C. Entropy-Mediated Patterning of Surfactant-Coated Nanoparticles and Surfaces. Phys. Rev. Lett. 2007, 99 (22), 226106.
Egorov, S. a. Microphase Separation of Mixed Polymer Brushes Physisorbed on Cylindrical Surfaces. Soft Matter 2012, 8 (14), 3971.

Vilain, C.; Goettmann, F.; Moores, A.; Le Floch, P.; Sanchez, C. Study of Metal Nanoparticles Stabilised by Mixed Ligand Shell: A Striking Blue Shift of the Surface-Plasmon Band Evidencing the Formation of Janus Nanoparticles. J. Mater. Chem. 2007, 17 (33), 3509-3514.

Nel, A. E.; Mädler, L.; Velegol, D.; Xia, T.; Hoek, E. M. V.; Somasundaran, P.; Klaessig, F.; Castranova, V.; Thompson, M. Understanding Biophysicochemical Interactions at the NanoBio Interface. Nat. Mater. 2009, 8 (7), 543-557.

Lundqvist, M.; Stigler, J.; Elia, G.; Lynch, I.; Cedervall, T.; Dawson, K. A. Nanoparticle Size and Surface Properties Determine the Protein Corona with Possible Implications for Biological Impacts. Proc. Natl. Acad. Sci. 2008, 105 (38), 14265-14270.

Xia, X.-R.; Monteiro-Riviere, N. A.; Riviere, J. E. An Index for Characterization of Nanomaterials in Biological Systems. Nat. Nanotechnol. 2010, 5 (9), 671-675.

Penna, M.; Ley, K.; Maclaughlin, S.; Yarovsky, I. Surface Heterogeneity: A Friend or Foe of Protein Adsorption - Insights from Theoretical Simulations. Faraday Discuss. 2016, 191, 435-464.

Ong, Q.; Luo, Z.; Stellacci, F. Characterization of Ligand Shell for Mixed-Ligand Coated Gold Nanoparticles. Acc. Chem. Res. 2017, 50 (8), 1911-1919.

Li, T.; Senesi, A. J.; Lee, B. Small Angle X-Ray Scattering for Nanoparticle Research. Chem. Rev. 2016, 116 (18), acs.chemrev.5b00690.

Kim, B. H.; Shin, K.; Kwon, S. G.; Jang, Y.; Lee, H.-S.; Lee, H.; Jun, S. W.; Lee, J.; Han, S. Y.; Yim, Y.-H.; et al. Sizing by Weighing: Characterizing Sizes of Ultrasmall-Sized Iron Oxide Nanocrystals Using MALDI-TOF Mass Spectrometry. J. Am. Chem. Soc. 2013, 135 (7), 2407-2410.

Liu, X.; Deng, R.; Zhang, Y.; Wang, Y.; Chang, H.; Huang, L.; Liu, X. Probing the Nature of Upconversion Nanocrystals: Instrumentation Matters. Chem. Soc. Rev. 2015, 44 (6), 14791508.

Azubel, M.; Koivisto, J.; Malola, S.; Bushnell, D.; Hura, G. L.; Koh, A. L.; Tsunoyama, H.; Tsukuda, T.; Pettersson, M.; Hakkinen, H.; et al. Electron Microscopy of Gold Nanoparticles at Atomic Resolution. Science (80-. ). 2014, 345 (6199), 909912.

Mancini, G. F.; Latychevskaia, T.; Pennacchio, F.; Reguera, J.; Stellacci, F.; Carbone, F. Order/Disorder Dynamics in a Dodecanethiol-Capped Gold Nanoparticles Supracrystal by Small-Angle Ultrafast Electron Diffraction. Nano Lett. 2016, 16 (4), 2705-2713.

Lee, Z.; Jeon, K.-J.; Dato, A.; Erni, R.; Richardson, T. J.; Frenklach, M.; Radmilovic, V. Direct Imaging of Soft-Hard Interfaces Enabled by Graphene. Nano Lett. 2009, 9 (9), 33653369.

Marbella, L. E.; Millstone, J. E. NMR Techniques for Noble Metal Nanoparticles. Chem. Mater. 2015, 27 (8), 2721-2739. Gentilini, C.; Franchi, P.; Mileo, E.; Polizzi, S.; Lucarini, M.; Pasquato, L. Formation of Patches on 3D SAMs Driven by Thiols with Immiscible Chains Observed by ESR Spectroscopy. Angew. Chemie Int. Ed. 2009, 48 (17), 3060-3064.

Lucarini, M.; Pasquato, L. ESR Spectroscopy as a Tool to Investigate the Properties of Self-Assembled Monolayers Protecting Gold Nanoparticles. Nanoscale 2010, 2 (5), 668. Şologan, M.; Marson, D.; Polizzi, S.; Pengo, P.; Boccardo, S.; Pricl, S.; Posocco, P.; Pasquato, L. Patchy and Janus Nanoparticles by Self-Organization of Mixtures of Fluorinated and Hydrogenated Alkanethiolates on the Surface of a Gold Core. ACS Nano 2016, 10 (10), 9316-9325.

Pengo, P.; Şologan, M.; Pasquato, L.; Guida, F.; Pacor, S.; Tossi, A.; Stellacci, F.; Marson, D.; Boccardo, S.; Pricl, S.; et al. Gold Nanoparticles with Patterned Surface Monolayers for Nanomedicine: Current Perspectives. Eur. Biophys. J. 2017, 46 (8), 749-771.

Jackson, A. M.; Hu, Y.; Silva, P. J.; Stellacci, F. From Homoligand- to Mixed-Ligand- Monolayer-Protected Metal 
Nanoparticles: A Scanning Tunneling Microscopy Investigation. J. Am. Chem. Soc. 2006, 128 (34), 11135-11149.

(34) Ong, Q. K.; Reguera, J.; Silva, P. J.; Moglianetti, M.; Harkness, K.; Longobardi, M.; Mali, K. S.; Renner, C.; De Feyter, S.; Stellacci, F. High-Resolution Scanning Tunneling Microscopy Characterization of Mixed Monolayer Protected Gold Nanoparticles. ACS Nano 2013, 7 (10), 8529-8539.

(35) Schreiber, F. Self-Assembled Monolayers: From "simple" Model Systems to Biofunctionalized Interfaces. J. Phys. Condens. Matter 2004, 16 (28), R881-R900.

(36) Rabanel, J.-M.; Adibnia, V.; Tehrani, S. F.; Sanche, S.; Hildgen, P.; Banquy, X.; Ramassamy, C. Nanoparticle Heterogeneity: An Emerging Structural Parameter Influencing Particle Fate in Biological Media? Nanoscale 2019, 11 (2), 383-406.

(37) Luo, Z.; Marson, D.; Ong, Q. K.; Loiudice, A.; Kohlbrecher, J.; Radulescu, A.; Krause-Heuer, A.; Darwish, T.; Balog, S.; Buonsanti, R.; et al. Quantitative 3D Determination of SelfAssembled Structures on Nanoparticles Using Small Angle Neutron Scattering. Nat. Commun. 2018, 9 (1), 1343.

(38) Luo, Z.; Zhao, Y.; Darwish, T.; Wang, Y.; Hou, J.; Stellacci, F. Mass Spectrometry and Monte Carlo Method Mapping of Nanoparticle Ligand Shell Morphology. Nat. Commun. 2018, 9 (1), 4478

(39) Liu, X.; Yu, M.; Kim, H.; Mameli, M.; Stellacci, F. Determination of Monolayer-Protected Gold Nanoparticle Ligand-Shell Morphology Using NMR. Nat. Commun. 2012, 3 (1182), 1-9.

(40) Moglianetti, M.; Ong, Q. K.; Reguera, J.; Harkness, K. M.; Mameli, M.; Radulescu, A.; Kohlbrecher, J.; Jud, C.; Svergun, D. I.; Stellacci, F. Scanning Tunneling Microscopy and Small Angle Neutron Scattering Study of Mixed Monolayer Protected Gold Nanoparticles in Organic Solvents. Chem. Sci. 2014, 5 (3), 1232.

(41) Centrone, A.; Hu, Y.; Jackson, A. M.; Zerbi, G.; Stellacci, F. Phase Separation on Mixed-Monolayer-Protected Metal Nanoparticles: A Study by Infrared Spectroscopy and Scanning Tunneling Microscopy. Small 2007, 3 (5), 814-817.

(42) Riccardi, L.; Gabrielli, L.; Sun, X.; De Biasi, F.; Rastrelli, F.; Mancin, F.; De Vivo, M. Nanoparticle-Based Receptors Mimic Protein-Ligand Recognition. Chem 2017, 3 (1), 92-109.

(43) Anand, U.; Lu, J.; Loh, D.; Aabdin, Z.; Mirsaidov, U. Hydration Layer-Mediated Pairwise Interaction of Nanoparticles. Nano Lett. 2016, 16 (1), 786-790.

(44) Dahal, U.; Wang, Z.; Dormidontova, E. E. Hydration of Spherical PEO-Grafted Gold Nanoparticles: Curvature and Grafting Density Effect. Macromolecules 2018, 51 (15), 59505961.

(45) Pensado, A. S.; Pádua, A. A. H. Solvation and Stabilization of Metallic Nanoparticles in Ionic Liquids. Angew. Chemie Int. Ed. 2011, 50 (37), 8683-8687.

(46) Svergun, D. I.; Koch, M. H. J.; Timmins, P. A.; May, R. P. Small Angle X-Ray and Neutron Scattering from Solutions of Biological Macromolecules; Oxford University Press, 2013.

(47) Chu, B.; Liu, T. Characterization of Nanoparticles by Scattering Techniques. J. Nanoparticle Res. 2000, 2 (1), 29-41.

(48) Disch, S.; Wetterskog, E.; Hermann, R. P.; Wiedenmann, A.; Vainio, U.; Salazar-Alvarez, G.; Bergström, L.; Brückel, T. Quantitative Spatial Magnetization Distribution in Iron Oxide Nanocubes and Nanospheres by Polarized Small-Angle Neutron Scattering. New J. Phys. 2012, 14 (1), 013025.

(49) Jia, H.; Grillo, I.; Titmuss, S. Small Angle Neutron Scattering Study of Polyelectrolyte Brushes Grafted to Well-Defined Gold Nanoparticle Interfaces. Langmuir 2010, 26 (10), 7482-7488.

(50) Von White, G.; Mohammed, F. S.; Kitchens, C. L. Small-Angle Neutron Scattering Investigation of Gold Nanoparticle Clustering and Ligand Structure Under Antisolvent Conditions. J. Phys. Chem. C 2011, 115 (38), 18397-18405.

(51) Diroll, B. T.; Weigandt, K. M.; Jishkariani, D.; Cargnello, M.; Murphy, R. J.; Hough, L. A.; Murray, C. B.; Donnio, B. Quantifying "Softness" of Organic Coatings on Gold Nanoparticles Using Correlated Small-Angle X-Ray and Neutron Scattering. Nano Lett. 2015, 15 (12), 8008-8012. (52) Rahme, K.; Oberdisse, J.; Schweins, R.; Gaillard, C.; Marty, J.D.; Mingotaud, C.; Gauffre, F. Pluronics-Stabilized Gold
Nanoparticles: Investigation of the Structure of the PolymerParticle Hybrid. Chemphyschem 2008, 9 (15), 2230-2236.

(53) Kim, S.; Kim, T. H.; Huh, J.; Bang, J.; Choi, S. H. Nanoscale Phase Behavior of Mixed Polymer Ligands on a Gold Nanoparticle Surface. ACS Macro Lett. 2015, 4 (4), 417-421. Yoonessi, M.; Seikel, E.; Pender, M. J. Characterization and Modeling of Stable Colloids of Organically Surface Tailored Gold Nanoparticle Liquids. Langmuir 2009, 25 (6), 3369-3373. Hore, M. J. A.; Ye, X.; Ford, J.; Gao, Y.; Fei, J.; Wu, Q.; Rowan, S. J.; Composto, R. J.; Murray, C. B.; Hammouda, B. Probing the Structure, Composition, and Spatial Distribution of Ligands on Gold Nanorods. Nano Lett. 2015, 15 (9), 57305738 .

(56) Zheng, N.; Fan, J.; Stucky, G. D. One-Step One-Phase Synthesis of Monodisperse Noble-Metallic Nanoparticles and Their Colloidal Crystals. J. Am. Chem. Soc. 2006, 128 (20), 6550 6551.

(57) Luo, Z.; Hou, J.; Menin, L.; Ong, Q. K.; Stellacci, F. Evolution of the Ligand Shell Morphology during Ligand Exchange Reactions on Gold Nanoparticles. Angew. Chemie - Int. Ed. 2017, 56 (43), 13521-13525.

(58) Franke, D.; Petoukhov, M. V.; Konarev, P. V.; Panjkovich, A.; Tuukkanen, A.; Mertens, H. D. T.; Kikhney, A. G.; Hajizadeh, N. R.; Franklin, J. M.; Jeffries, C. M.; et al. ATSAS 2.8: A Comprehensive Data Analysis Suite for Small-Angle Scattering from Macromolecular Solutions. J. Appl. Crystallogr. 2017, 50 (4), 1212-1225.

(59) Svergun, D. I. Restoring Low Resolution Structure of Biological Macromolecules from Solution Scattering Using Simulated Annealing. Biophys. J. 1999, 76 (6), 2879-2886.

(60) Hansen, J.-P.; Hayter, J. B. A Rescaled MSA Structure Factor for Dilute Charged Colloidal Dispersions. Mol. Phys. 1982, 46 (3), 651-656.

(61) Rambo, R. P.; Tainer, J. A. Super-Resolution in Solution X-Ray Scattering and Its Applications to Structural Systems Biology SAXS: Small-Angle X-Ray Scattering. Annu. Rev. Biophys 2013, 42, 415-441.

(62) Smith, A. M.; Marbella, L. E.; Johnston, K. A.; Hartmann, M. J.; Crawford, S. E.; Kozycz, L. M.; Seferos, D. S.; Millstone, J. E. Quantitative Analysis of Thiolated Ligand Exchange on Gold Nanoparticles Monitored by ${ }^{1} \mathrm{H}$ NMR Spectroscopy. Anal. Chem. 2015, 87 (5), 2771-2778.

(63) Radulescu, A.; Szekely, N. K.; Appavou, M.-S. KWS-2: Small Angle Scattering Diffractometer. J. large-scale Res. Facil. JLSRF 2015, 1, A29.

(64) Edwards, W.; Marro, N.; Turner, G.; Kay, E. R. Continuum Tuning of Nanoparticle Interfacial Properties by Dynamic Covalent Exchange. Chem. Sci. 2018, 9 (1), 125-133.

(65) Bradford, S. M.; Fisher, E. A.; Meli, M. V. Ligand Shell Composition-Dependent Effects on the Apparent Hydrophobicity and Film Behavior of Gold Nanoparticles at the Air-Water Interface. Langmuir 2016, 32 (38), 9790-9796.

(66) Kuna, J. J.; Voïtchovsky, K.; Singh, C.; Jiang, H.; Mwenifumbo, S.; Ghorai, P. K.; Stevens, M. M.; Glotzer, S. C.; Stellacci, F. The Effect of Nanometre-Scale Structure on Interfacial Energy. Nat. Mater. 2009, 8 (10), 837-842.

(67) Harkness, K. M.; Balinski, A.; McLean, J. A.; Cliffel, D. E. Nanoscale Phase Segregation of Mixed Thiolates on Gold Nanoparticles. Angew. Chemie Int. Ed. 2011, 50 (45), 1055410559.

(68) Farrell, Z.; Merz, S.; Seager, J.; Dunn, C.; Egorov, S.; Green, D. L. Development of Experiment and Theory to Detect and Predict Ligand Phase Separation on Silver Nanoparticles. Angew. Chemie - Int. Ed. 2015, 54 (22), 6479-6482.

(69) Centrone, A.; Penzo, E.; Sharma, M.; Myerson, J. W.; Jackson, A. M.; Marzari, N.; Stellacci, F. The Role of Nanostructure in the Wetting Behavior of Mixed-Monolayer-Protected Metal Nanoparticles. Proc. Natl. Acad. Sci. 2008, 105 (29), 98869891.

(70) Pedersen, J. S.; Posselt, D.; Mortensen, K.; Jan, B. Y.; Pedersen, S.; Posselt, D.; Mortensen, K. Analytical Treatment of the Resolution Function for Small-Angle Scattering. J. Appl. Crystallogr. 1990, 23 (4), 321-333. 



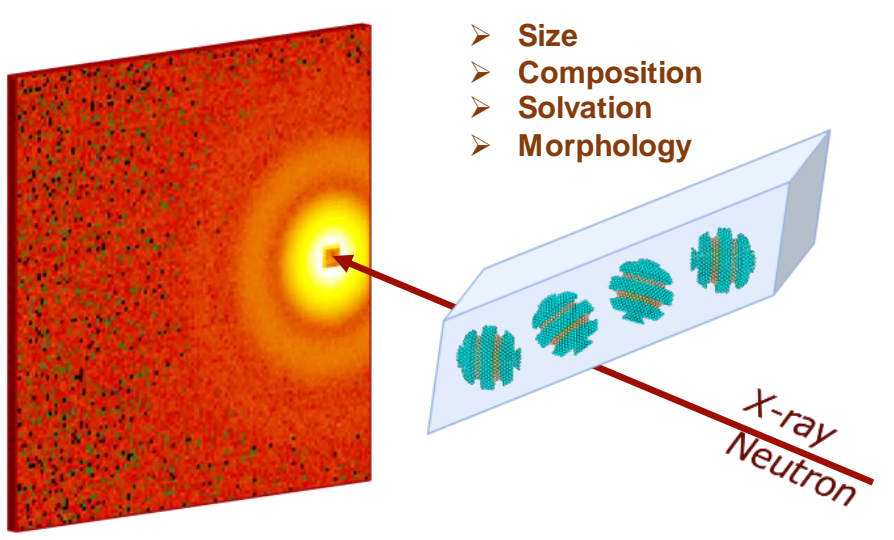

Table of Contents artwork 